\title{
A New Mathematical Model of Exception Handling in Java Object Oriented Language
}

\author{
Manoj Kumar Srivastav \\ Indira Gandhi National Open University \\ St. Xavier's College (Autonomous), Kolkata, Pin \\ Code-700016, \\ India
}

\author{
Asoke Nath, Ph.D \\ Associate Professor, Department of Computer \\ Science, St. Xavier's College \\ (Autonomous),Kolkata, Pin code-700016, India
}

\begin{abstract}
Srivastav et al already published several papers where the authors tried to explain the various aspects of object oriented language like Java using some simple mathematical model or functions. The authors have already studied the general properties of Java language such as object, class, inheritance, multithreaded concepts using simple mathematical concepts. In the present paper the authors have tried to explore the concept of exception handling in java language using discontinuity in a function. The exceptions are normally come during runtime and some of the exceptions are repairable and some may not be repairable. In the present paper the authors tried to explain both repairable exception and also irreparable exceptions by considering simple continuity and discontinuity in functions.
\end{abstract}

\section{Keywords}

Exception handling, discontinuity ,function, continuity, object

\section{INTRODUCTION}

Compilation is a process that translates a program in one language (the source language) into an equivalent program in another language (the object or target language). An important role of any compiler is the detection and reporting of errors. The source code is a high-level programming language which may be $\mathrm{C}$ or $\mathrm{C}++$ or $\mathrm{C} \#$ or Java or any other language. The target code may be machine language code or assembly code. Thus compilation is a fundamental concept in the production of software: it is the link between the (abstract) world of application development and the low-level world of application execution on machines. Functions are the major tools for describing the real world in mathematical terms. A function plays a very important role in the programming language like $\mathrm{C}$, Java etc. The set of all possible input values is called the domain of the function. The set of all output values is called the range of the function. In calculus , Leohard Euler (1707-1783) invented a symbolic way to say " $y$ is function of $\mathrm{x}$ " i.e. $\mathrm{y}=\mathrm{f}(\mathrm{x})$ (" $\mathrm{y}$ equals $\mathrm{f}$ of $\mathrm{x}$ ").In this notation, the symbol ' $\mathrm{f}$ " represents the function. The letter $\mathrm{x}$, called the independent variable, represent an input value from the domain of $\mathrm{f}$, and $\mathrm{y}$, the dependent variable, represent the corresponding output values $f(x)$ in the range of $f$.

The authors have found a close relationship between mathematical functions and exceptional handling. There are two types of mathematical function :(i)Continuous function and (ii)Discontinuous function. The source function in a programming language may be continuous or Discontinuous function. When the source function in the programming language, either in procedural language like $\mathrm{C}$ or in object oriented language like Java, is continuous there is no interruption in the flow of the program but on the other hand if the source function is discontinuous function, there will be an interruption in the programs that is called runtime error. In procedural language there is no way to prevent run time errors as there is no concept called exception handling. The advantage of object oriented programming language like Java is that one can trap the runtime errors using exception handling routines which is not there in so called procedural language like "C".

\section{MATHEMATICAL FUNCTION THAT ARE GENERALLY TAKEN AS SOURCE FUNCTION/ VALUE}

Real -Valued Functions : A function $\mathrm{f}: \mathrm{X} \quad \mathrm{Y}$ is a correspondence(or a rule) that assigns to each $\mathrm{x} \varepsilon \mathrm{X}$, a unique elements $y=f(x)$ in $Y$. $X$ is called the domain of $f$ and $Y$, the co-domain of $f$. The unique element $y=f(x)$ in $Y$, that is assigned to $x$, by the function $f$ is called the value of $f$ at $x$. The symbol $\mathrm{x}$, that take the values from the dom $\mathrm{f}$, is called the independent variable, while the symbol y to denote $f(x)$, is called the dependent variable.

The subset $f(X)=\{y \mid y=f(x), x \in X\}$ is subset of $Y$, is called the range of $f$.

If $\mathrm{Y} \subseteq \mathrm{R}$ then the function $\mathrm{f}: \mathrm{X} \quad \mathrm{Y}$, takes up real value only .For this reason, it is called a real-valued function or simply a real function.

If $\mathrm{X} \subseteq \mathrm{R}$, then the independent variable also takes up real value only. In this case, the function $\mathrm{f}: \mathrm{X} \quad \mathrm{Y}$ is called a real function of real variable.

Some Elementary Real Function:

(i)A constant function $\mathrm{f}_{\mathrm{c}}: \mathrm{f}_{\mathrm{c}}(\mathrm{x})=\mathrm{c}$ for all $\mathrm{x} \varepsilon \mathrm{R} \operatorname{dom} \mathrm{f}_{\mathrm{c}}=\mathrm{R}$, $\mathrm{f}_{\mathrm{c}}(\mathrm{R})=\{\mathrm{c}\}$

(ii)The identity function, $\mathrm{I}: \mathrm{I}(\mathrm{x})=\mathrm{x}$, for all $\mathrm{x} \varepsilon \mathrm{R}$, dom $\mathrm{I}=\mathrm{R}$, $\mathrm{I}(\mathrm{R})=\mathrm{R}$.

(iii)Reciprocal function $\mathrm{f}: \mathrm{f}(\mathrm{x})=1 / \mathrm{x}$, for all $\mathrm{x} \varepsilon(\mathrm{R}-\{0\})$, dom $\mathrm{f}=\mathrm{R}-\{0\}, \mathrm{f}(\mathrm{R}-\{0\})=\mathrm{R}$

(iv)Absolute value function' ||$^{\prime}:|x|=x$, if $x>0$ and $|x|=-x$ if $\mathrm{x}<0$

(v)Positive integral Power function

$, \mathrm{x}^{\mathrm{n}}: \mathrm{x} \longrightarrow \mathrm{x}^{\mathrm{n}} \mathrm{x} \varepsilon \mathrm{R}, \mathrm{n}$ a positive integer. $\operatorname{dom} \mathrm{x}^{\mathrm{n}}=\mathrm{R}, \operatorname{ran} \mathrm{x}^{\mathrm{n}}=$ $\mathrm{R}$, if $\mathrm{n}$ is odd And $\operatorname{ran} \mathrm{x}^{\mathrm{n}}=\mathrm{R}^{+} \cup\{0\}$, if $\mathrm{n}$ is even.

(vi)Polynomial Function $P_{n}: P_{n}(x)=a_{0} x^{n}+a_{1} x^{n-1}+a_{2} x^{n-}$ ${ }^{2}+\ldots \ldots+a_{n}$ Where $a_{0}, a_{1}, a_{2}, \ldots \ldots \ldots, a_{n}$ are constants, $a_{0} \neq 0 ; n$ a positive integer $\operatorname{dom} \mathrm{P}_{\mathrm{n}}=\mathrm{R}, \quad \operatorname{ran} \mathrm{P}_{\mathrm{n}} \subseteq \mathrm{R}$

(vii)Rational function, $\mathrm{R}: \mathrm{R}(\mathrm{x})=\mathrm{P}(\mathrm{x}) / \mathrm{Q}(\mathrm{x}), \mathrm{x} \in \mathrm{R} \quad \mathrm{Q}(\mathrm{x}) \neq 0$, $\mathrm{P}(\mathrm{x}), \mathrm{Q}(\mathrm{x})$ are polynomial.dom ' $\mathrm{R}$ ' $=\mathrm{R}-\{\mathrm{x} \mid \mathrm{Q}(\mathrm{x})=0\}$, ran ' $\mathrm{R}$ ' $\subseteq \mathrm{R}$ 
(viii)Bracket function, [.]:[x]=greatest integer not greater than $\mathrm{x}, \mathrm{x} \& \mathrm{R}$

dom'[ ]' $=\mathrm{R}$, ran'[ ]'=Z

(ix)qth root function, $\mathrm{x}^{1 / \mathrm{q}:} \mathrm{y}=\mathrm{x}^{1 / \mathrm{q}}$ iff $\mathrm{y}^{\mathrm{q}}=\mathrm{x}, \mathrm{q}$ is a positive integer.

dom' $x^{1 / q}$ ‘ $=R^{+}\{0\}$, if $q$ is even

dom' $x^{1 / q}=R$, if $q$ is odd. $\quad \operatorname{ran}$ ' $x^{1 / q} \subseteq R$.

(x)The sin function, sin : dom sine $=\mathrm{R}$ ran sine $=[-1,1]$.

$\sin (-\mathrm{x})=-\sin \mathrm{x}$ for all $\mathrm{x} \varepsilon \mathrm{R}$

$\sin (2 \pi+x)=\sin x$ for all $x \in R$

$\sin (\mathrm{A}+\mathrm{B})=\sin \mathrm{A} \cos \mathrm{B}+\cos \mathrm{A} \sin \mathrm{B}$, for all $\mathrm{A}, \mathrm{B} \varepsilon \mathrm{R}$

(xi)The cosine function, cos : dom cosine $=\mathrm{R}$, for cosine $=[-$ $1,1]$.

$\cos (-\mathrm{x})=\cos \mathrm{x}$ for all $\mathrm{x} \varepsilon \mathrm{R}$

$\cos (2 \pi+x)=\cos x$ for all $x \in R$

$\cos (\mathrm{A}+\mathrm{B})=\cos \mathrm{A} \cos \mathrm{B}-\sin \mathrm{A} \sin \mathrm{B}$, for all $\mathrm{A}, \mathrm{B} \varepsilon \mathrm{R}$

(xii) Exponential function, $\mathrm{e}^{\mathrm{x}}$ :

$\mathrm{e}^{\mathrm{x}}=1+\mathrm{x}+\mathrm{x}^{2} / 2 !+\ldots \ldots+\mathrm{x} / \mathrm{n} !+\ldots \ldots .$.

$\operatorname{dom} \mathrm{e}^{\mathrm{x}}=\mathrm{R}, \operatorname{ran}^{\mathrm{x}}=(0, \infty)$

$\mathrm{e}^{\mathrm{x}+\mathrm{y}}=\mathrm{e}^{\mathrm{x}} \cdot \mathrm{e}^{\mathrm{y}}$ and $\mathrm{e}^{-\mathrm{x}}=1 / \mathrm{e}^{-\mathrm{x},} \mathrm{x}, \mathrm{y} \in \mathrm{R}$

(xiii) Logarithm function, $\log \mathrm{x}$ : $\mathrm{y}=\log \mathrm{x}$ iff $\mathrm{e}^{\mathrm{y}}=\mathrm{x}, \mathrm{x}>0$

dom $\log \mathrm{x}=\mathrm{R}^{+,}$ran $\log \mathrm{x}=\mathrm{R}$

$\log (\mathrm{xy})=\log \mathrm{x}+\log \mathrm{y}, \log (1 / \mathrm{x})=-\log \mathrm{x}, \mathrm{x}, \mathrm{y} \varepsilon \mathrm{R}^{+}$

(xiv) Inverse sine (or arc sine), $\sin ^{-1}$ :

$\mathrm{y}=\sin ^{-1} \mathrm{x} \quad$ iff $\mathrm{x}=\sin \mathrm{y}$

dom $\sin ^{-1}=[-1,1]$, ran $\sin ^{-1}=[-\pi / 2, \mathrm{c} \pi / 2]$

(xv) Inverse cosine (or arc cosine function), $\cos ^{-1}$ :

$\mathrm{y}=\cos ^{-1} \mathrm{x}$ iff $\mathrm{x}=\cos \mathrm{y}$

dom $\cos ^{-1}=[-1,1]$, ran $\cos ^{-1-}=[0, \pi]$

\section{MATHEMATICAL DESCRIPTION OF THE EXCEPTIONAL HANDLING IN JAVA}

The exception handling in java is one of the powerful mechanism to handle the runtime errors so that normal flow of the application can be maintained. Dictionary Meaning of Exception is an abnormal condition .In java, exception is an event that disrupts the normal flow of the program. It is an object which is thrown at runtime. Similarly, a function $f(x)$ is commonly said to be continuous provided its graph is a continuous curve, and if there is any discontinuity or break at any point on the curve, the function is said to be discontinuous for the corresponding value of $x$. The general notations of continuity of a function $\mathrm{f}(\mathrm{x})$ for any value of the variable $\mathrm{x}$ requires that the function should be finite at that point, and for very small change in $x$, the change in the value of $f(x)$ should be small, or in other words as we approach the particular value of $x$ from either side the function should also approach the corresponding value of $\mathrm{f}(\mathrm{x})$, and ultimately coincide with it at that point. If $\mathrm{f}(\mathrm{x})$ be non-existent at a point, so the corresponding point on the graph is missing, or else, if the value of $f(x)$ suddenly jumps as $x$ passes from one side to the other or the particular value, or $f(x)$ becomes infinitely large at a point, then the function is discontinuous there. In the object- oriented programming language, if we define a mapping $\mathrm{f}:(\mathrm{X}, \boldsymbol{\tau}) \quad \rightarrow \quad(\mathrm{Y}, \sigma)$ such that $\mathrm{f}$ (address of object $)=$ address of new object $[$ Here $X$ and $Y$ are object set and are $\tau$ set of sets with respect to object $X$ and $\sigma$ are set of sets with respect to object $\mathrm{Y}$.Actually represents classes for domain and represents class for range in the above function and $f$ represents main function.] then for each open subset $V$ of $Y$,the set $f^{-1}(V)$ is open subset of $X$. Since Exception is an object which is thrown at run time. So the concept of continuity and discontinuity may be apply only on the the object i.e., simply on the sets only.

Intuitively, a function is continuous at $x=c$ if we can draw it without lifting our pen from the paper. Formally, a function is continuous at $\mathrm{x}=\mathrm{c}$ on the open interval $(\mathrm{a}, \mathrm{b})$ if the following three conditions are met :

$$
\begin{aligned}
& \text { (i) } \mathrm{f}(\mathrm{c}) \text { is defined } \\
& \text { (ii) } \lim _{\mathrm{f}}(\mathrm{x}) \text { exists } \\
& \mathrm{x} \rightarrow \mathrm{c} \\
& \text { (iii) } \lim _{\mathrm{f}} \mathrm{x}(\mathrm{x})=\mathrm{f}(\mathrm{c}) \\
& \mathrm{x} \rightarrow c
\end{aligned}
$$

If $\mathrm{f}$ be a function defined in an interval $\mathrm{I}$ and $\mathrm{x}_{0} \varepsilon \mathrm{I}, \mathrm{f}$ is said to be discontinuous at $x_{0}$, if it is not continuous at $x_{0}$. Also in that case , the point $\mathrm{x}_{0}$ is said to be a power of discontinuity or simply a discontinuity of $\mathrm{f}$.

(a) Types of Discontiuity:

(i)Removable Discontinuity : Let $\mathrm{f}$ be a function defined on an interval I and $\mathrm{p} \varepsilon$ I. If

$\lim f(x)$ exists but not equal $f(p)$, then $p$ is called

$\mathrm{x} \rightarrow \mathrm{p}$

a removable discontinuity of $f$.

(ii)Discontinuity of first kind: Let $\mathrm{f}$ be a function defined on an interval I and $\mathrm{p} \varepsilon$ I. If

$f(p-0)$ and $f(p+0)$ both exists but are unequal, then $p$ is called discontinuity of the first kind.

If $f(p)=f(p+0)$, but $f(p-0) \neq f(p)$, then $p$ is called a discontinuity of first kind from the left.

If $f(p-0)=f(p)$, but $f(p) \neq f(p+0)$, then $p$ is called a discontinuity of first kind from the right

(iii)Discontinuity of second kind : Let $f$ be a function defined on an interval I and $\mathrm{p} \varepsilon$ I. If neither $\mathrm{f}(\mathrm{p}-0)$ and $\mathrm{f}(\mathrm{p}+0)$ exists, then $p$ is called a discontinuity of the second kind of $f$

If one of the two one-sided limits of $f, f(p-0)$ or $f(p+0)$ exists while the other does not, then $p$ is called a point of mixed discontinuity or discontinuity of $f$ of second kind, from the left or the right as the case may be.

(b)Types of Exception in object Oriented Programming(in Java)

There are mainly two types of exceptions: checked and unchecked. The exception error considered as unchecked exception. The sun micro system says there are three types of exceptions:

\section{(i)Checked Exception}

(ii)Unchecked Exception

(iii)Error 


\section{(i) Checked Exception}

The classes that extend Throwable class except Runtime Exception and Error are known as checked exceptions e.g.IOException, SQLException etc. Checked exceptions are checked at compile-time.

\section{(ii)Unchecked Exception}

The classes that extend Runtime Exception are known as unchecked exceptions e.g. Arithmetic Exception, NullPointerException, ArrayIndexOutOfBoundsException etc. Unchecked exceptions are not checked at compile-time rather they are checked at runtime.

\section{(iii)Error}

Error is irrecoverable e.g. OutOfMemoryError, VirtualMachineError, AssertionError etc.

The purpose of Exceptional handling mechanism is to provide a means to detect and report an "exceptional circumstance" so that appropriate action be taken. The mechanism suggests incorporation of separate error handling code that performs the following tasks:

(a)Find the problem (Hit the Exception).

(b)Inform that an error has occurred(Throw the exception)

(c)Receive the error information(Catch the exception)

(d)Take corrective actions(Handle the exception)

The error handling code basically consists of two segments, one to detect errors and to throw exceptions and the other to catch exception and to take appropriate actions. Similarly Removal discontinuity is also a discontinuous function .But it can be made to continuous at the discontinuous points by redefining its point at that point .Exceptional handling is also treating that function /value which are undefined. Therefore there is need of redefining the function using some special coding in exceptional handling .

\section{SOME EXAMPLE OF FUNCTION WHERE REMOVAL DISCONTINUITY OCCURS ON DISCONTINOUS FUNCTION}

When a graph is drawn for a function, there may exist hole in a graph .i.e if there exist a hole in the graph, the given function is discontinuous .Therefore, discontinuity of the function can be repaired by filling a single point In other words, intituively, removable discontinuity is a point at which a graph is not connected but can be made connected by filling in a single point.Formally, a removable discontinuity is one at which the limit of the function exists but does not equal the value of the function at that point; this may be because the function does not exist at that point.

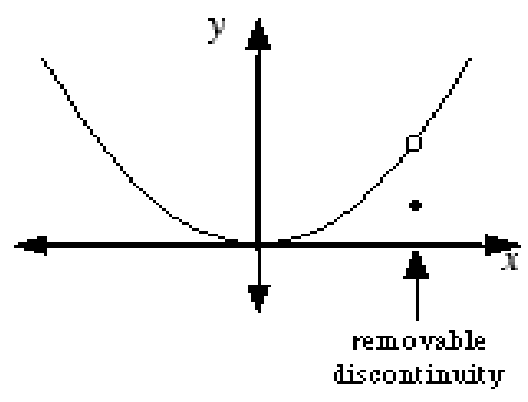

Figure -1 For removable discontinuity
The set of points of removable discontinuities of a function is countable.

Example 1: If the function factors and the bottom term cancels, the discontinuity at the $x$-value for which the denominator was zero is removable, so the graph has a hole in it.

Consider, the function $\mathrm{f}: \mathrm{X} \longrightarrow \mathrm{R}$

$\mathrm{f}(\mathrm{x})=\left(\mathrm{x}^{2}-4 \mathrm{x}-21\right) /(\mathrm{x}+3)$

$=((\mathrm{x}+3) *(\mathrm{x}-7)) /(\mathrm{x}+3)$ for $\mathrm{x}$ belongs to set $\mathrm{X}$ and here $\mathrm{R}$ is representing set of real numbers.

After canceling, it leaves with $x-7$. Therefore $x+3=0$ (or $x$ $=-3$ ) is a removable discontinuity - the graph has a hole, as shown in figure a.
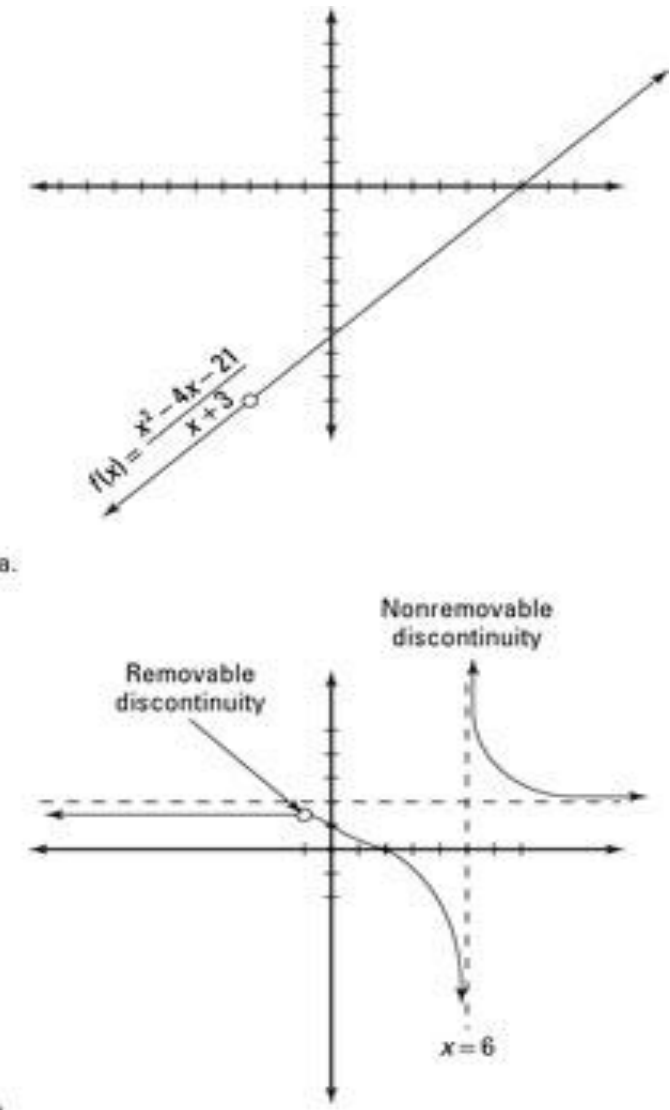

Example 2 :If a term doesn't cancel, the discontinuity at this $x$ value corresponding to this term for which the denominator is zero is non -removable, and the graph has a vertical asymptote.

Consider a function $\mathrm{g}: \mathrm{X} \longrightarrow \mathrm{R}$

The following function factors as shown:

$$
\begin{aligned}
\mathrm{g}(\mathrm{x}) & =\left(\mathrm{x}^{2}-\mathrm{x}-2\right) /\left(\mathrm{x}^{2}-5 \mathrm{x}-6\right) \\
& =((\mathrm{x}+1) *(\mathrm{x}-2)) /((\mathrm{x}+1) *(\mathrm{x}-6))
\end{aligned}
$$

for $\mathrm{x}$ belongs to set $\mathrm{X}$ and here $\mathrm{R}$ is representing set of real number. Because the $x+1$ cancels, there is a removable discontinuity at $x=-1$ (i.e, there is a hole in the graph, not an asymptote). But the $x-6$ didn't cancel in the denominator, so, there is a nonremovable discontinuity at $x=6$. This discontinuity creates a vertical asymptote in the graph at $x=6$. The figure $\mathrm{b}$ above shows the graph of $g(x)$ 


\section{SOME EXAMPLE OF FUNCTION WHERE EXCEPTION OCCURS ON SOURCE FUNCTION \\ Common scenarios where exceptions may} occur

There are given some scenarios where unchecked exceptions can occur. They are as follows:

(i) Scenario where ArithmeticException occurs

If any number is divided by zero, there occurs an ArithmeticException.

int $\mathrm{a}=50 / 0 ; / /$ ArithmeticException

//exception_1.java: Write a program to test number format

//exception

import java.io.*;

class exception_1

\{ IOException

public static void main(String args[])throws

BufferedReader $\quad$ br=new $\quad$ BufferedReader(new InputStreamReader(System.in));

int i,nvalid,ninvalid,num, $a=5, b=6, c=6, d$;

nvalid $=$ ninvalid $=0$

for $(\mathrm{i}=0 ; \mathrm{i}<\operatorname{args}$. length; $\mathrm{i}++)$

\{

try

num=Integer.parseInt(args[i]);

$\mathrm{d}=\mathrm{a} /(\mathrm{b}-\mathrm{c})$;

nvalid=nvalid +1 ;

Number="+num);

System.out.println("InValid

catch(ArithmeticException e)

System.out.println("\nDivide by zeroln");

\}

catch(NumberFormatException e)

\{

ninvalid=ninvalid +1

System.out.println("\nInvalid

number="+args[i]);

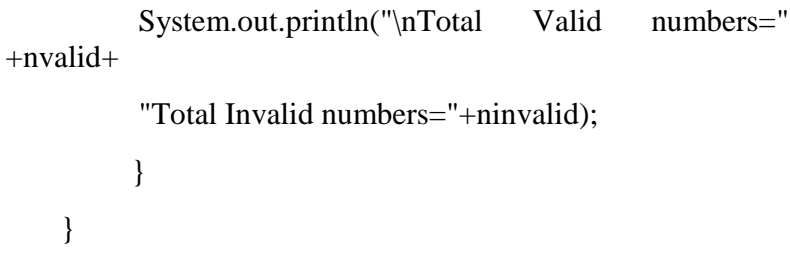

\begin{tabular}{|l|l|}
\hline $\begin{array}{l}\text { Exceptional } \\
\text { Handling }\end{array}$ & $\begin{array}{l}\text { Removable } \\
\text { Discontinuity/ condition }\end{array}$ \\
\hline $\mathrm{a}=5, \mathrm{~b}=6, \mathrm{c}=6$ & $\begin{array}{l}\text { Consider a function } \\
\mathrm{f}(\mathrm{x})=5 /(6-\mathrm{x})\end{array}$ \\
$\begin{array}{l}\text { ArithmeticException } \\
\mathrm{e}\end{array}$ & $\begin{array}{l}\text { This function is } \\
\text { continuous at all points of } \\
\text { real numbers except at } \\
\mathrm{x}=6,\end{array}$ \\
& $\begin{array}{l}\text { So for this function e } \\
\text { removable condition } \\
\text { may be taken as } \\
\mathrm{f}(\mathrm{x})=5 /(6-\mathrm{x}) \quad=\mathrm{c} \quad \mathrm{a} \\
\text { constant value at } \mathrm{x}=6\end{array}$ \\
\hline
\end{tabular}

(ii)Scenario where NumberFormatException occurs The wrong formatting of any value, may occur NumberFormatException. Suppose there is a string variable that has characters, converting this variable into digit will occur

NumberFormatException.

String s="abc";

int $\mathrm{i}=$ Integer.parseInt(s);//NumberFormatException

//except_1.java : program to check number format import java.io.*;

class except_1

\{

public static void main(String args[])throws IOException

\section{\{}

int inv_count,vld_count, i;

int n,number;

$\mathrm{n}=\operatorname{args}$.length;

inv_count=vld_count $=0$;

for $(\mathrm{i}=0 ; \mathrm{i}<\mathrm{n} ; \mathrm{i}++)$

\{

try

\{

number=Integer.parseInt(args[i]);

\}

catch(NumberFormatException e)

\{

inv_count++;

System.out.println("Invalid

number="+args[i]); 
continue;

\}

System.out.println("Valid number="+args[i]);

vld_count++;

$$
\text { \} }
$$

System.out.println("Valid numbers="+vld_count);

System.out.println("Invalid numbers="+inv_count); \}

\}

\begin{tabular}{|c|c|}
\hline Exceptional Handling & $\begin{array}{l}\text { Removable } \\
\text { Discontinuity/con } \\
\text { dition }\end{array}$ \\
\hline $\begin{array}{l}\text { The wrong formatting of } \\
\text { any value, may occur } \\
\text { NumberFormatException. } \\
\text { Suppose there is a string } \\
\text { variable that have } \\
\text { characters, converting this } \\
\text { variable into digit will occur } \\
\text { NumberFormatException. } \\
\text { String s="abc"; } \\
\text { int i=Integer.parseInt(s);//N } \\
\text { umberFormatException }\end{array}$ & 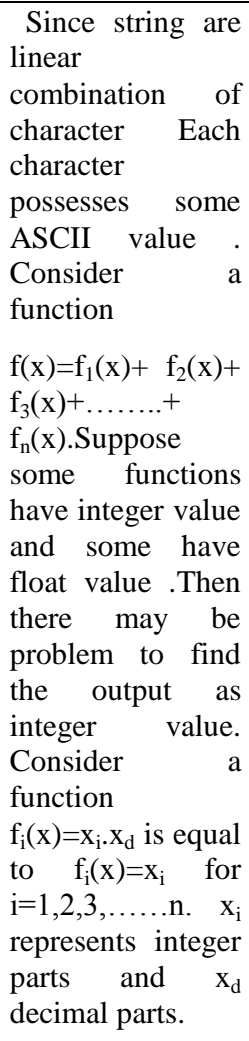 \\
\hline
\end{tabular}

(iii) Scenario where ArrayIndexOutOfBoundsException occurs

The ArrayIndexOutOfBoundsException will arise if the index of the array is taken some invalid value such as negative or may be more than the upper limit of the array. An example is given below:

int $\mathrm{a}[]=$ new int [5];

$\mathrm{a}[10]=50$; //ArrayIndexOutOfBoundsException

//xxexception_3.java: Write a program to use ArrayStoreExecption to implement when wrong data type is introduced in an array import java.io.*;

class xxexception_3

\{ IOException

public static void main(String args[])throws

\{

int $\mathrm{a}[]=$ new int[5];

int i,n,s,m;

BufferedReader br=new BufferedReader(new InputStreamReader(System.in));

System.out.print("InEnter size of $\operatorname{array}(1-5):$ :");

$\mathrm{n}=$ Integer.parseInt(br.readLine());

$\mathrm{s}=0$;

$\mathrm{m}=0$;

for $(\mathrm{i}=0 ; \mathrm{i}<\mathrm{n} ; \mathrm{i}++)$

\{

try

\{

System.out.print("a["+i+"]=");

$\mathrm{a}[\mathrm{i}]=$ Integer.parseInt(br.readLine());

$\mathrm{m++}$;

$\mathrm{s}=\mathrm{s}+\mathrm{a}[\mathrm{i}]$;

\}

catch(NumberFormatException e)

\{

typeln");

System.out.println("'InEntered a wrong data

\}

catch(ArrayIndexOutOfBoundsException e)

\{

System.out.println("InIndex of Array is out of

Rangeln");

\}

\}

System.out.println("Sum of "+m+" numbers="+s);

\} 


\begin{tabular}{|c|c|}
\hline Exceptional Handling & Mathematical Description \\
\hline $\begin{array}{l}\text { If the index of an array is invalid then it would result } \\
\text { an error ArrayIndexOutOfBoundsException as } \\
\text { shown below: } \\
\text { int a[]=new int [5]; } \\
\text { a[10]=50; //ArrayIndexOutOfBoundsException } \\
\text { ArrayIndexOutOfBoundsException e) }\end{array}$ & $\begin{array}{l}\text { Consider a function } \mathrm{f}(\mathrm{x})=\sin \mathrm{x} \text { for } \mathrm{x} \varepsilon[-\pi / 2, \pi / 2] \\
\text { This function is continuous in this interval. Suppose } \\
\text { if someone wants to find the value at } \mathrm{x}=\pi \text {, then this } \\
\text { value is not lies in domain }[-\pi / 2, \pi / 2] \text { of } \mathrm{f}(\mathrm{x}) \text {. }\end{array}$ \\
\hline
\end{tabular}

However, it is not possible to change all the discontinuous functions to continuous functions by removing some condition. Similarly, it is not possible to implement all possible exceptions in object oriented programming language In the table below a comparative is made among exceptional handling, cause of exceptions and the mathematical descriptions

\begin{tabular}{|c|c|c|}
\hline .Exceptional Handling & Cause of Exception & Mathematical Description \\
\hline FileNotFoundException & $\begin{array}{l}\text { Caused by an attempt to access } \\
\text { a non- existent file }\end{array}$ & $\begin{array}{l}\text { Files are stream of characters. } \\
\text { Characters makes one to one } \\
\text { correspondence with the ASCII } \\
\text { value. ASCII values are subset } \\
\text { of real numbers. } \\
\text { Consider a function like } \\
\text { func }(\mathrm{x})=1 / \mathrm{x} \text {, the func }(\mathrm{x}) \text { does } \\
\text { not exist at } \mathrm{x}=0 \text {, so to overcome } \\
\text { this difficulty the following } \\
\text { condition may be taken: } \\
\mathrm{f}(\mathrm{x})=1 / \mathrm{x}, \mathrm{x} \neq 0 \\
\quad=0, \mathrm{x}=0 \text {. }\end{array}$ \\
\hline IOException & $\begin{array}{l}\text { Caused by general } \mathrm{I} / \mathrm{O} \text { failures } \\
\text { such as inability to read from a } \\
\text { file. These are the signals that } \\
\text { an I/O exception of some sort } \\
\text { has occurred }\end{array}$ & $\begin{array}{l}\text { In the some composite function } \\
\text { (like product/division) is made } \\
\text { in such way that individual is } \\
\text { continuity is not considered. Let } \\
\mathrm{f}(\mathrm{x})=\mathrm{x}^{2} \sin (1 / \mathrm{x}), \mathrm{x} \neq 0 \\
\quad=0, \mathrm{x}=0 \text {. } \\
\text { is a continuous function where } \\
\text { individual discontinuity is not } \\
\text { taken. }\end{array}$ \\
\hline
\end{tabular}

\begin{tabular}{|c|c|c|}
\hline OutofMemoryException & $\begin{array}{l}\text { Caused when there is not } \\
\text { enough memory to allocate a } \\
\text { new object. }\end{array}$ & $\begin{array}{l}\text { Let } \mathrm{I}=[\mathrm{a}, \mathrm{b}] \text { be a closed and } \\
\text { bounded interval and let } \\
\text { function } \mathrm{f}: \mathrm{I} \underset{\mathrm{R}}{\mathrm{R}} \mathrm{be} \\
\text { continuous on I. Then } \mathrm{f} \text { is } \\
\text { bounded on I.Also there is a } \\
\text { point ce[a,b] such that } \\
\mathrm{f}(\mathrm{c})=\operatorname{supf}(\mathrm{x}) \\
\quad \mathrm{x} \varepsilon \mathrm{I} \\
\text { and also there is a point } \\
\mathrm{d} \varepsilon[\mathrm{a}, \mathrm{b}] \text { such that } \mathrm{f}(\mathrm{d})=\inf \mathrm{f}(\mathrm{x}) \\
\mathrm{x} \varepsilon \mathrm{I}\end{array}$ \\
\hline StackOverFlowException & $\begin{array}{l}\text { Caused when the system } \\
\text { runs out of stack space }\end{array}$ & $\begin{array}{l}\text { Let a function } \mathrm{g}: \mathrm{R} \longrightarrow \mathrm{R} \\
\text { is unbounded on every } \\
\text { neighbourhood of a point } \\
\text { ceR. In that case } \mathrm{g} \text { is } \\
\text { discontinuous at } \mathrm{c} \text { whether } \mathrm{g}\end{array}$ \\
\hline
\end{tabular}




\begin{tabular}{|l|l|l|}
\hline StringIndexOutofBoundsException & $\begin{array}{l}\text { Caused when a program } \\
\text { attempts to access a } \\
\text { nonexistent character } \\
\text { position a string. }\end{array}$ & $\begin{array}{l}\text { This type of case is } \\
\text { repairable. There is not any } \\
\text { impact on the function even } \\
\text { if there is not one-one to } \\
\text { mapping between index set } \\
\text { and functional value. }\end{array}$ \\
\hline
\end{tabular}

(iv)Handling of some exception with mathematical explanation

\begin{tabular}{|c|c|}
\hline Exception syntax & Mathematical Explanation \\
\hline $\begin{array}{l}\text { Syntax of try with catch block } \\
\operatorname{try}\{ \\
\ldots \\
\text { \}catch(Exception_class_Name reference })\{\}\end{array}$ & $\begin{array}{l}\qquad \text { If } \lim \mathrm{f}(\mathrm{x}) \quad=\mathrm{L} \text { but } \mathrm{f}(\mathrm{a}) \\
\mathrm{x}-->\mathrm{a} \\
\text { is not defined } \\
\text { then the discontinuity at } \mathrm{x}=\mathrm{a} \text { can be removed by } \\
\text { defining } \mathrm{f}(\mathrm{a})=\mathrm{L}\end{array}$ \\
\hline $\begin{array}{l}\operatorname{try}\{ \\
\cdots \\
\text { \}finally }\{\}\end{array}$ & $\begin{array}{l}\text { When a finally block is defined, this is guaranteed } \\
\text { to execute, regardless of whether or not in } \\
\text { exception is thrown. There is little change in the } \\
\text { mathematics, which states that " if } \mathrm{f} \text { is uniformly } \\
\text { continuous on an interval I then it is necessarily } \\
\text { continuous on I whereas converse may not be } \\
\text { true." }\end{array}$ \\
\hline $\begin{array}{l}\text { Multiple catch block: } \\
\text { If we have to perform different tasks at the } \\
\text { occurrence } \\
\text { Of different Exceptions, use multiple catch block. } \\
\text { Syntax: try }\{ \\
\ldots \\
\text { \}catch(Exception_class1_Name reference) }\{\} \\
\{ \\
\text { Statement } \\
\} \text { catch(Exception_class-n_Name reference) }\{\}\end{array}$ & $\begin{array}{l}\text { If it is possible to remove the discontinuity at each } \\
\text { discontinuous point we have to remove the } \\
\text { discontinuity at each point respectively one by one }\end{array}$ \\
\hline 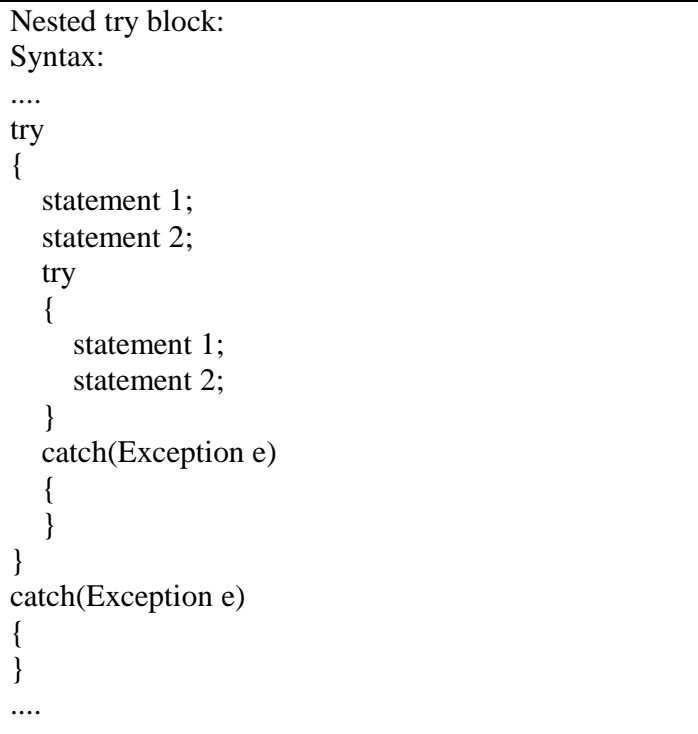 & $\begin{array}{l}\text { We know that composition of a continuous } \\
\text { function is continuous function .so to check the } \\
\text { continuity of function fl(f } 2(\mathrm{f} 3 \ldots . . .(\mathrm{fn}))) \text {, it is } \\
\text { required to check the continuity of } \mathrm{f} \text { first and if it } \\
\text { is continuous then o.k. otherwise it is required to } \\
\text { remove its discontinuity. Next we have to check } \\
\text { the continuity of } \mathrm{f}(\mathrm{n}-1) \text { and apply the same } \\
\text { process and so on and lastly we check the } \\
\text { continuity of f1. }\end{array}$ \\
\hline
\end{tabular}

\section{CONCLUSION AND FUTURE SCOPE}

The core advantage of exception handling is to maintain the normal flow of the application. Exception normally disrupts the normal flow of the application that is why se exception handling use. The future scope of Exceptional Handling lies that one can find different method /coding to handle different discontinuous functions. It can be used to evaluate integration, Riemann integration, It is known that every continuous function is integrable but converse may not be true. Riemann Integral also deals with discontinuous functions. There is lots of scope to find different 
methodologies to get the output of different integrable function .It is still believed that the graph of the function could be traced with a" free motion of hand", i.e function were still believed to be piecewise smooth. So, there is scope to think from continuous and discontinuous function for development of exceptional handling in java.

\section{REFERENCES}

[1] Manoj Kumar Srivastav , Asoke Nath Mathematical modeling of various statements of C-type Language, , International Journal of Advanced Computer Research(IJACR), Vol-3,Number-1, Issue-13, Page:7987 Dec(2013).

[2] Manoj Kumar Srivastava, Asoke Nath Mathematical Description of variables, pointers, structures, Unions used in C-type language, , Joiurnal of Global Research Computer Science, Vol-5, No-2, Page:24-29, Feb(2014)

[3] Manoj Kumar Srivastav, Asoke Nath, A Mathematical Modeling of Object Oriented Programming Language : a case study on Java programming language, Current Trends in Technology and Science(CTTS) Vol-3, Issue3, Page 134-141,(2014).

[4] Manoj Kumar Srivastav, Asoke Nath, Analysis of Compilation Errors, Runtime Errors, Reliability and Validity of a Program: A Case Study on C-language : IJISET - International Journal of Innovative Science, Engineering \& Technology, Vol. 1 Issue 3, May 2014. Page-281-288.

[5] Mathematical foundations of Multithreaded programming concepts in Java language,Manoj Kumar Srivastav, Dr. Asoke Nath, International Journal of Innovative Research in Information Security(IJIRIS)Volume1 Issue4(October2014)Page 6-17

[6] E Balaguruswamy. 2011- Programming with JavaTataMcGrawHill EductionPrivate Limited.
[7] Sachin Malhotra, Sourabh Choudhary.2012 Programming in Java- OXFORD University PressHerbert Schildt,2001 Java TM 2: The Complete Reference, TataMcGraw-Hill Publishing Company Limited,

[8] Satish Jain,Vineeta Pillai,Kratika.2011 Introduction to Object Oriented Programming through Java,BPB Publications

[9] S.K.Mapa, 1998 Real Analysis, Asoke Prakasan

[10] S.K.Mapa,2000 Higher Algebra, -Sarat Book Distribution,

[11] K.D.Joshi-Topology.New Age International Publiocation.

[12] J.N Sharma.2000 "Topology", Krishna Prakashan Media(p)Ltd., Meerut,

[13] R.S.aggarwal.2000,Topology-S.chand publication.

[14] James R Munkers. 2001,Topology- Pearson Education Asia,

[15] www.javatpoint.com

[16] R.D.Sharma, 2011, Mathematics class xi-. Danapat Rai Publications(P) LTD.

[17] Inder K. Rana. 1999 An Introduction to measure and integration- Narosa publication House

[18] Tom M. Apostal. 1997 Mathematical Analysis- Narosa Publishing House

[19] http://www.mathwords.com/r/removable_discontinuity.h tm

[20] http://hungry4java.blogspot.in/2014_07_01_archive.html

[21] S.C.Malik,SavitaArora .2006 Mathematical Analysis", New Age International(P) Limited Publishers 